\title{
Endotype Driven Treatment of Asthma
}

\section{Endotypes and Asthma Treatment}

\section{Ioana Octavia Agache, MD, PhD}

\author{
Address \\ Department of Allergy and Clinical Immunology, Transylvania University, \\ Spatarul Luca Arbore 16, Brasov, Romania 500112 \\ Email: ibrumaru@unitbv.ro
}

Published online: 15 March 2014

(C) Springer International Publishing AG 2014

Keywords Asthma - Endotype - Targeted treatment - Biomarkers - Inflammation - Remodeling

\section{Opinion Statement}

The heterogeneity of asthma in relation to clinically significant outcomes, including response to treatment, has been established beyond any doubt. However, current treatment guidelines for asthma ignore disease heterogeneity and causal pathways. Extended heterogeneous disease-related metabolic, inflammatory, immunological, and remodeling pathways have been described, and a repetitive pattern is defined as a disease endotype. The response to targeted and non-targeted interventions in asthma may vary among individuals or for the same individual in relation to outcome measures (dissociated effect). Targeted treatment should be both biomarker-driven and outcome-driven. The ideal biomarker should be pathway-specific, reproducible, easily measurable, and affordable. Biomarker research in asthma is increasingly shifting from the assessment of the value of single biomarkers to multidimensional approaches, in which the clinical value of a combination of various markers is studied. Translation of biomarkers into pathway-specific diagnostic tests is essential and should guide the design of future large clinical trials, incorporating both longitudinal and mechanism-tailored endpoints. The selection of outcome measure is difficult, as it must reflect the mechanistic intervention and should be relevant for both the asthmatic population in general and the particular individual with asthma. While endotype-driven therapeutic strategies are increasingly successful, the issues of dissociated effect and drug efficacy at the target site remain unresolved. Efforts needed to move the field forward include profiling of Th2low inflammation, incorporation of new targets, such as airway smooth muscle and epithelial components of asthma or epigenetics modifications, as well as application of systems pharmacology.

\section{Introduction}

It has recently become increasingly evident that antiasthmatic drugs are more effective in relation to certain molecular mechanisms of asthma, and hence the emergence of biomarkers to predict response to treatment. 
Data have suggested that sputum eosinophils are accurate predictors for inhaled corticosteroids (ICS) response, both in corticosteroid-naïve patients and in cases of moderate/severe asthma, while neutrophilic inflammation was associated with lack of response. Other data sets have shown that the baseline values of clinical parameters, particularly lung function, are the major predictors of ICS response, and that blood or sputum eosinophils merely complement this data [1-5]. In addition, several reports have questioned the stability of the sputum eosinophilic phenotype over time, especially in the pediatric population $[6$, 7]. High levels of fractional exhaled NO (FeNO) have also been suggested as predictive of response to ICS, although the tailoring of treatment based on FeNO measurements did not decrease asthma exacerbations or increase asthma control [8]. There are several factors that influence FeNO levels, including age, atopy, medication use, and airway infections, and these must be considered when using FeNO for tailored interventions.

The accuracy of FeNO level surrogate for eosinophilic inflammation has been questioned in recent studies $[9,10]$. One study that examined the use of blood eosinophils, FeNO, FEV1, and IgE levels, either alone or in combination, found that these were not accurate predictors of sputum eosinophilia [11]. A longitudinal study that assessed the relationship between sputum eosinophils and FeNO in children with asthma produced variable results in almost half of the subjects who produced more than one sputum sample, and it was not possible to identify a group in whom
FeNO would consistently reflect eosinophilia [12]. The lack of correlation can be explained if we accept that $\mathrm{FeNO}$ and blood eosinophils reflect different endotypes of Th2-mediated inflammation. In a crosssectional study, FeNO and blood eosinophil values offered independent information with respect to the prevalence of wheeze, asthma diagnosis, and asthma events [13•]. The authors suggest that blood eosinophilia is a marker of more severe systemic inflammation driven by a strong chemokine signal (such as IL$5)$ and more extensive eosinophilic airway inflammation involving the small airways, and therefore is ICS non-responsive. It may also highlight the risk of asthma exacerbations requiring oral corticosteroids. Further evidence was provided in the DREAM trial, which indicated that blood eosinophils were most closely related to a positive response to mepolizumab

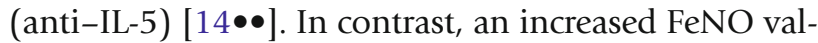
ue indicated IL-4/IL-13-mediated Th2 inflammation localized in the bronchial mucosa responding to ICS or to IL-4/IL-13 blockade. Increased FENO value was a good predictor of a clinical response to lebrikizumab in the MILLY trial $[15 \bullet \bullet]$.

Exhaled air metabolomics was recently evaluated as a predictor of ICS response. In patients with mild/moderate asthma, breath analysis by eNose predicted response with greater accuracy than sputum eosinophils or FeNO [16]. Studies are needed, however, to validate the clinical relevance of volatile organic compounds (VOCs), as well as longitudinal assessment of VOC patterns, in a large population of asthmatic patients.

\section{A new concept: endotype-driven treatment of asthma}

The hallmark characteristics of asthma, including inflammation, remodeling, and airway hyperreactivity, are governed by a complex network of molecules, sometimes repetitive across individuals with asthma. Although endotypes for asthma have been proposed, to date, no asthma endotypes have been definitively validated $[17 \bullet, 18 \bullet]$. The PRACTALL consensus report proposed several parameters for defining an asthma endotype: consistent clinical characteristics, biomarkers, lung physiology, genetic background, histopathology, epidemiology, and treatment response [19•]. The identification of corresponding molecular biomarkers for the individual pathogenic mechanisms underlying phenotypes or subgroups within a phenotype is essential (Fig. 1, Table 1). Longitudinal studies are necessary to validate whether newly defined asthma endotypes predict the individual course of the disease [18 $\bullet$. 


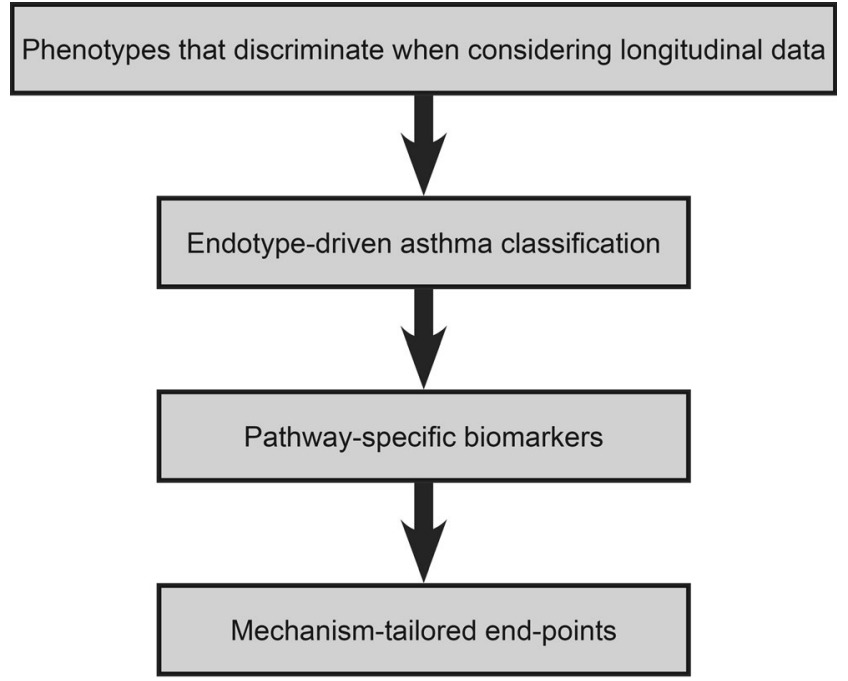

Fig. 1. Essential steps to improve response to asthma treatment.

Unfortunately, asthma endotyping has focused primarily on Th2-high inflammation, which accounts for only half of the cases, while airway smooth muscle (ASM) and epithelial components and the Th2-low inflammation have been neglected $[17 \bullet]$.

\section{The Th2-high asthma endotype}

In one study, the Th2-high molecular phenotype of asthma was described based on expression of periostin, human calcium-activated chloride channel 1 (CLCA1), and serpinB2, and characterized by increased expression of IL-5 and IL-13 in bronchial biopsies, airway hyperresponsiveness (AHR), serum IgE, blood and airway eosinophilia, subepithelial fibrosis, and airway mucin gene expression. The Th2 markers were reproducible on repeated evaluation. Response to ICS in the study was restricted to Th2-high asthma [20]. In a separate study, a qPCR-based assay of Th2 inflammation in bronchial biopsies was designed to overcome the limitations of the microarray-based method. The three-gene-mean of periostin, CLCA1, and serpinB2 correlated with FeNO, blood eosinophils, and PC20 methacholine, with greater improvement in FEV1 under ICS, and was a better predictor of improvement of lung function and symptoms than FeNO, blood eosinophils, IgE, or PC20 [21]. In induced sputum cell pellets, PCR was used to profile the gene expression of the epithelial cell signature of IL-13 activation and the Th2 genes as a noninvasive measure of Th2 inflammation. Gene expression levels of CLCA1 and periostin, but not SerpinB2, were significantly higher in sputum cells from asthmatics. Expression of IL-4, IL-5, and IL-13 was also significantly increased and highly correlated within individual subjects. By combining the expression levels of Th2 genes in a single quantitative metric (Th2 


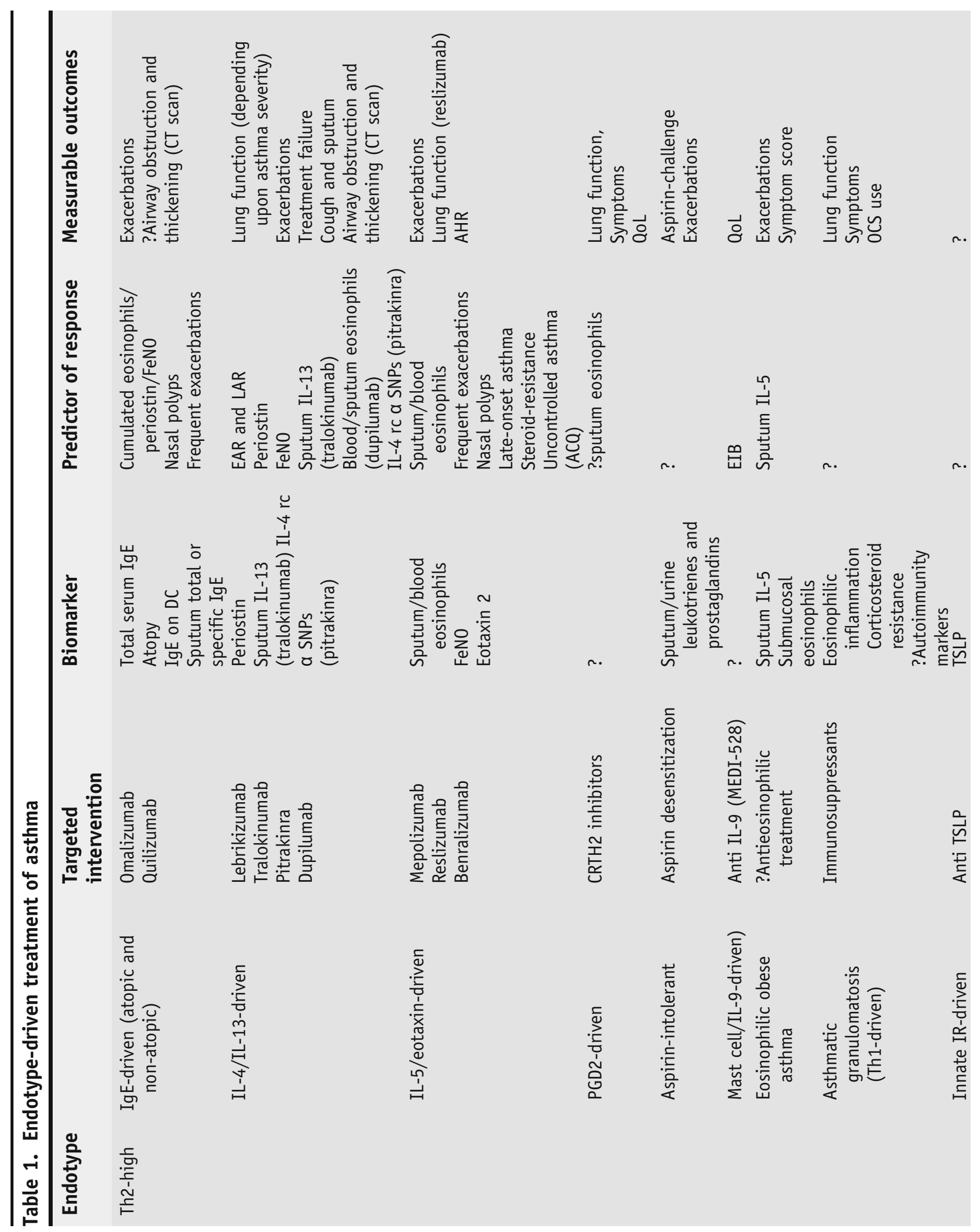




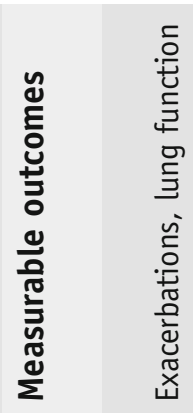

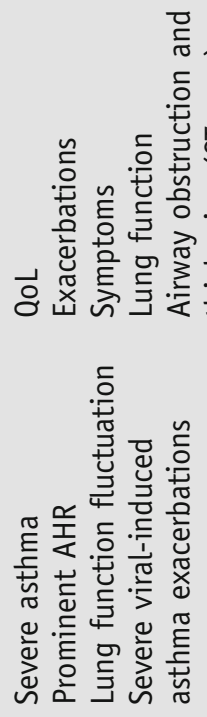

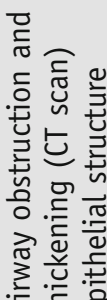

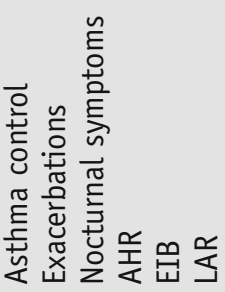

돈.

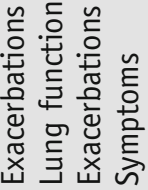

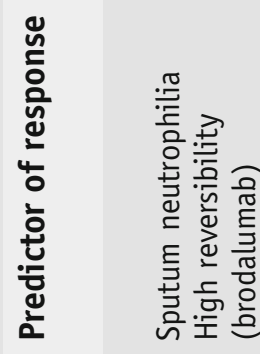
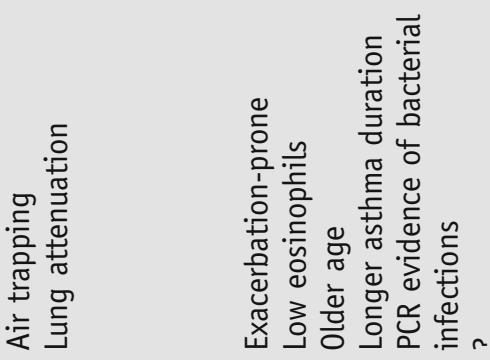

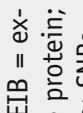

岂

它总范

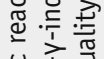

는

ज胥

근은

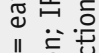

㟧离

宛.

空

范赑

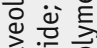

항응

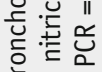

II 离范

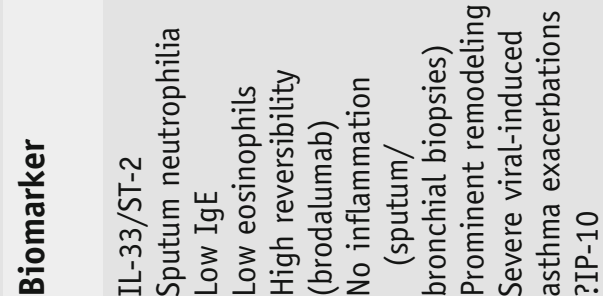

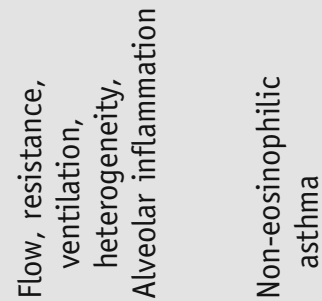

丕

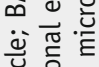

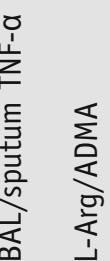

寒莣

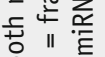

员垴宅

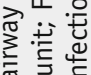

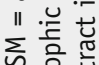

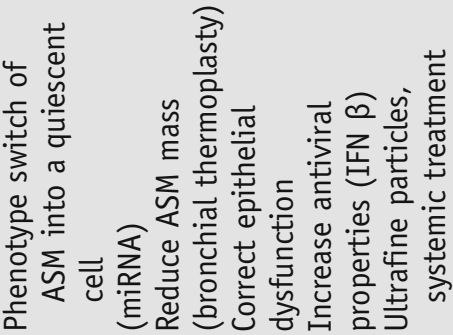

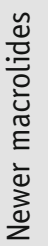

竞 ?

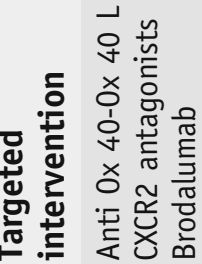

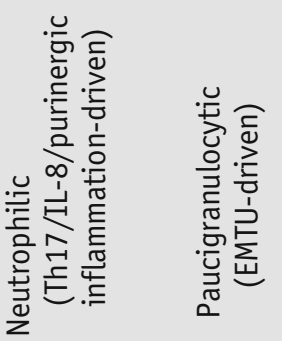

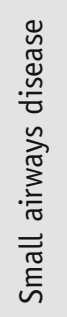

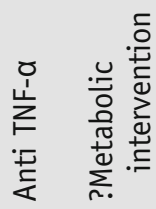

总部言

J

흘

를 II

学完

II 흥

足

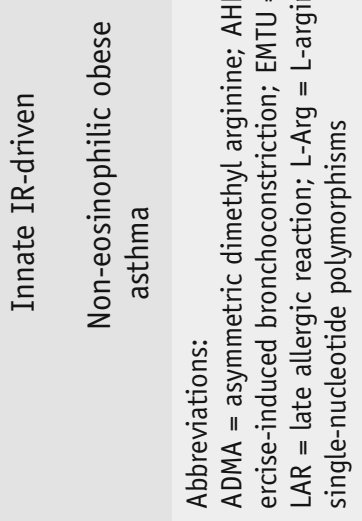


gene mean), $70 \%$ had Th2-high asthma, which was characterized by increased asthma severity and blood and sputum eosinophilia [22].

Several subtypes of the Th2-high endotype can be described based on the main operating molecular mechanism (Table 1).

Omalizumab, an anti-IgE monoclonal antibody, has been found to have significant benefits for patients with moderate-to-severe uncontrolled allergic asthma, although predictors of response are limited. In the Inner City Asthma Study (ICAS), both sensitivity and exposure were better predictors of response [23]. A post hoc analysis of the EXTRA study confirmed the potential of Th2 biomarkers (FeNO, blood eosinophils, and serum periostin) as baseline predictors for the therapeutic benefit of omalizumab [24•].

IgE has been linked to asthma irrespective of atopic status. In patients with refractory non-atopic asthma, omalizumab resulted in a statistically significant reduction in FceRI expression on basophils and plasmacytoid dendritic cells (pDC2). The omalizumab group showed an overall increase in FEV1 compared with baseline as well as a trend toward improvement in global evaluation of treatment effectiveness and asthma exacerbation rate [25•]. It is proposed that the mechanism is either targeting local IgE or balancing the innate immune response to multiple triggers of an exacerbation by decreasing FceRI expression on pDC [26]. In the Inner City Asthma Study, omalizumab nearly eliminated seasonal peaks (both spring and fall) in exacerbations, with or without viral infection, by targeting the cell (pDC) at the crossroads of asthma exacerbation triggers [23].

Sputum samples from patients with intrinsic asthma showed increased levels of both total IgE and Der p-specific IgE, which may represent a specific biomarker for selecting patients with intrinsic asthma for anti-IgEtargeted intervention [27]. In a randomized double-blind placebo-controlled study of allergic and non-allergic patients with nasal polyps and comorbid asthma, omalizumab had a beneficial effect on airway symptoms (nasal congestion, anterior rhinorrhea, loss of sense of smell, wheezing, and dyspnea) and quality-of-life scores, irrespective of the presence of allergy [28]. The association with nasal polyps may also pinpoint responders to omalizumab in non-atopic asthma.

\section{IL-4/IL-13-driven Th2-high endotype}

The study of Lebrikizumab in Adult Patients With Asthma Who Are Inadequately Controlled on Inhaled Corticosteroids (MILR1444A - MILLY trial) was one the first monoclonal antibody trials in asthma to validate a biomarker consistent with the therapeutic intervention. In this double-blind placebo-controlled 6-month trial in patients with inadequately controlled asthma, the IL-13 blocking antibody lebrikizumab significantly improved FEV1 at 12 weeks [15••]. A decrease in the rate of severe exacerbations at the 32-week follow-up period was also reported [29]. Patient subgroups were prespecified according to baseline total IgE level, blood eosinophil count, and serum periostin. The level of periostin was associated with a better response in terms of FEV1 improvement. In a post hoc analysis, high baseline $\mathrm{FeNO}$ was also associated with greater efficacy of lebrikizumab in improving 
lung function and decreasing severe exacerbations, although intrapatient variability was higher in baseline FeNO than in periostin levels during the run-in period. Adverse effects were similar to placebo, with the exception that musculoskeletal side effects occurred slightly more often with lebrikizumab. A dose-ranging study of lebrikizumab in adult patients not taking ICS (MOLLY trial) demonstrated no effect on FEV1 in this category of patients, including the periostin subgroup. However, lebrikizumab treatment was associated with a reduced risk of treatment failure at all doses, and results were similar in the periostin subgroup [30]. In a separate mild asthma trial, the late asthmatic response was reduced by $48 \%$ in lebrikizumab subjects, although this was not statistically significant. Exploratory analysis indicated a greater reduction in late asthmatic response in subjects with elevated baseline levels of peripheral blood eosinophils, serum IgE, or periostin [31].

Further studies are needed to explore the relationship between FeNO and periostin. In the MILLY trial, both were separate predictors of response. In the BOBCAT trial, serum periostin was strongly correlated with persistent sputum and tissue eosinophilia despite steroid treatment; the correlation was present regardless of sputum or tissue neutrophil counts. FeNO measurement detected fewer subjects with tissue eosinophilia and exhibited greater overlap between eosinophil-low and eosinophil-high subjects. While sputum and blood eosinophil counts and FeNO levels are subject to significant temporal variability based upon allergen exposure, exacerbations, and steroid treatment, the BOBCAT trial showed relatively little intra-subject variability in serum periostin in 3 measurements over the course of up to 5 weeks. In a logistic regression model incorporating age, sex, body mass index (BMI), blood eosinophils, serum IgE, FeNO, and serum periostin levels, periostin was the most significant single predictor of composite airway eosinophil status [32・].

Tralokinumab (CAT-354) is a human IgG4 monoclonal antibody that potently and specifically neutralizes IL-13. In a phase IIa study enrolling 194 patients with moderate-to-severe uncontrolled asthma despite controller therapies, tralokinumab significantly improved lung function and decreased rescue medication use, but did not affect the Asthma Control Questionnaire (ACQ)-6 score (primary outcome) or asthma exacerbations. The incidence of treatment-emergent AEs was higher in the tralokinumab groups than in the placebo group. Neither atopy nor blood eosinophils were better predictors for response. However, there was a trend for better response in FEV1 and ACQ-6 score the in the sputum IL-13-positive subjects [33].

The IL-4 receptor $\alpha$ amino acid variations may be another predictor of response to anti-IL-4/IL-13 pathway inhibitors. Subjects homozygous for the rs8832 common $G$ allele responded to pitrakinra administration with a decrease in exacerbations, nocturnal awakenings, and limitation of activities by asthma. Both rs8832 and rs1029489, in addition to several other intronic SNPs (rs3024585, rs3024622, and rs4787956), demonstrated a significant pitrakinra dose-response association with reduced asthma exacerbations. These SNPs were not associated with asthma exacerbations in the placebo group. The predictive power of SNPs, however, was present only in the loweosinophil-count group [34].

The efficacy and safety of dupilumab, a fully human monoclonal antibody to the $\alpha$-subunit of the interleukin- 4 receptor, was evaluated in pa- 
tients with uncontrolled persistent moderate-to-severe asthma who were preselected based on elevated blood or sputum eosinophil levels. The study evaluated the effects of dupilumab when added to background therapy, after long-acting acting beta- 2 agonists (LABA) discontinuation, during the tapering of ICS, and as monotherapy. Dupilumab was associated with fewer asthma exacerbations when LABAs and ICS were withdrawn and with improved lung function and reduced levels of Th2-associated inflammatory markers (FeNO, IgE, plasma eotaxin-3) [35•].

\section{IL-5 driven Th2-high endotype}

Mepolizumab, a humanized monoclonal antibody against IL-5, reduced the risk of asthma exacerbations in two small proof-of-concept studies. Patients with severe corticosteroid-resistant asthma were selected based on sputum eosinophilia and history of severe asthma exacerbations [36, 37]. The Dose Ranging Efficacy And safety with Mepolizumab in severe asthma (DREAM) trial tested the efficacy of mepolizumab for reducing the frequency of asthma exacerbations in severely asthmatic adults. The 621 adult subjects were selected based on history of severe exacerbations and evidence of eosinophilic inflammation, defined as either increased sputum or blood eosinophils, FeNO of $50 \mathrm{ppb}$ or more, or prompt deterioration of asthma control after a reduction of $25 \%$ or less in

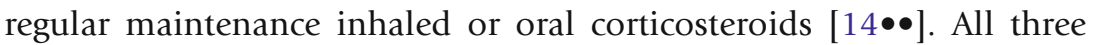
doses of intravenous mepolizumab significantly reduced exacerbations, delayed time to first exacerbation, and decreased exacerbations requiring hospitalization or ER visit. Both sputum and blood eosinophils were significantly reduced, although there was no change in lung function or quality-of-life (QoL) measures. Efficacy of mepolizumab increased with increased baseline eosinophil count and number of exacerbations in the previous year. Baseline FeNO proved to be less predictive than blood eosinophil count of response to treatment. The overall frequency of serious adverse events was similar across treatment groups.

Different results were obtained with reslizumab, another humanized monoclonal antibody anti-IL-5. In 53 subjects with persistent sputum eosinophilia and poorly controlled with ICS, reslizumab improved ACQ score only in patients with baseline ACQ scores $>2$ and those with nasal polyps. All patients in the reslizumab group showed a significant improvement in lung function, and sputum and blood eosinophils were reduced significantly. There was no effect on asthma exacerbations, likely due to the short duration of the study (15 weeks). Adverse events were similar to placebo [38].

\section{The Th2- low asthma endotype}

Based on data from Th2-high/low molecular signature studies, the incidence of Th2-low asthma accounts for 30-50\% of adult asthma cases [20-22]. Moderate or low response to ICS is characteristic, and a broad spectrum of asthma severity is included. Similar to the Th2-high endotype, several distinct subendotypes can be described (Table 1). 
Historically, anti-neutrophil agents such as anti-IL-8 have not met with much success. An orally active small-molecule antagonist of CXCR2 (SCH-527123) was recently tested in patients with severe asthma and sputum total cell count $<10 \times 10(6) / g$ and neutrophils $>40 \%$. SCH-527123 proved successful in reducing sputum neutrophil numbers, suggesting improvement in clinical outcomes such as mild exacerbations and ACQ scores. There was no effect on lung function or sputum neutrophil activation markers (myeloperoxidase, IL-8, or elastase). In terms of safety, the mean absolute neutrophil count in blood was reduced by $14 \%$ at the end of 4 weeks, but recovered by the 5 th week, and there were no differences in the overall rates of adverse events compared to placebo [39].

The efficacy and safety of brodalumab, a human anti-IL-17 receptor A monoclonal antibody, was recently evaluated in subjects with inadequately controlled moderate-to-severe asthma taking regular ICS. The primary endpoint was change in ACQ, and secondary endpoints included FEV1, symptom scores, and symptom-free days. Although there was no evidence of treatment of brodalumab in the overall study population, a high bronchodilator reversibility criteria (>20\%) identified a potential subpopulation for clinically meaningful response. Other prespecified subgroups (baseline FEV1\% predicted, ACQ, ICS dose, FeNO, peripheral eosinophils, sex, race and weight) did not predict response [40•]. Unfortunately, patients were not evaluated for their sputum inflammatory phenotype (eosinophilic or neutrophilic).

\section{Paucigranulocytic asthma (EMTU-driven endotype)}

A combination of studies on airway biopsies and primary cell cultures suggests that asthma is primarily an epithelial disease driven by increased environmental susceptibility to injury and an altered repair response as depicted by sustained activation of the epithelial mesenchymal trophic unit (EMTU) [41].

Significant advances in understanding the cell and molecular biology of inflammation and ASM contractility suggest an ASM asthmatic endotype and have identified several potential novel therapeutic targets [42]. Targeting G-protein-coupled receptors such as bitter taste receptors (TAS2R) may be an area of interest. In addition to the recently described bronchodilator and anti-inflammatory properties [43], their increased expression was shown in peripheral blood leucocytes of asthmatic children and in the airways of severe asthma patients $[44,45]$. TAS2R agonists were effective in relaxing ASM even when $\beta 2$-adrenergic receptors were subject to tachyphylaxis. In addition, IL-13 caused a decrease in $\beta$-agonist-mediated relaxation, while TAS2Rmediated relaxation was unaffected [46]. The cAMP/PKA pathway continues to be a promising drug target with the emergence of new phosphodiesteraseE inhibitors and a novel PKA target protein, HSP20, which mediates ASM relaxation via actin depolymerization. Inhibitors of the RhoA/Rho kinase pathway can also elicit ASM relaxation. Targeting epigenetic processes that control chromatin remodeling and RNA-induced gene silencing also holds great potential for "switching" of the ASM asthmatic endotype [42]. 
Ciliary dysfunction has been documented in moderate and severe asthma. The lack of correlation with eosinophilic inflammation indicates a distinct endotype associated with aberrant epithelial repair and infection [47]. Both short- and long-acting $\beta 2$-agonists are reported to increase ciliary beat frequency [48]. Therapy directed at ciliary dysfunction may represent a new treatment approach for this asthma endotype.

Lung epithelial cells can influence immune responses to allergens, viruses, and other triggers, as well as subsequent inflammation and repair. Barrier epithelial cells sense exposure via pattern recognition receptors and activate innate immune cells through the secretion of thymic stromal lymphopoietin (TSLP), GM-CSF, IL-1, IL-33, and IL-25 [41]. Inhibiting the IL-33/ST-2 axis may reduce the epithelial potential to induce a local Th2 orientation. TSLP is a potential noninvasive biomarker released from activated epithelial cells [49]. Specific humanized antibody that blocks interaction between TSLP and TSLP receptor or OX40 and OX40 ligand are currently being developed [50].

Airway epithelial cells are important initiators of the local antiviral immune response through the production of chemokines, proinflammatory cytokines, and interferons (IFN). An in vitro study showed that budesonide and formoterol can inhibit cell inflammatory responses in HBECs without interfering with viral replication or production of interferons [51]. Defective rhinovirus (RV)-induced IFN- $\beta$ and IFN- $\lambda$ production and increased RV replication have been reported in primary human bronchial epithelial cells (HBECs) from subjects with asthma [52]. In vitro addition of IFN- $\beta$ to HBEC restores the normal antiviral response [53]. Delivery of IFN- $\beta$ to the lungs of asthmatics may limit the spread of the virus to the lungs and consequent exacerbation. The phase II trial of inhaled IFN- $\beta$ (SNG 001) enrolled 134 adult asthmatics recruited during a cold episode. Patients taking IFN- $\beta$ showed lower asthma symptoms, a $65 \%$ reduction in exacerbations, and quicker lung function recovery. The response was significant only in patients with difficult-to-treat asthma [54].

\section{Microbiome-driven Th2-low asthma phenotype}

Several studies have reported the distortion in airway bacterial community structure and composition in asthma, with greater abundance of members of the Proteobacteria, particularly Haemophilus species, and lesser abundance of members of the Bacteroidetes, particularly Prevotellaceae $[55,56]$. Disordered microbiome was related to greater AHR and corticosteroid resistance [57, $58 \bullet]$.

Two approaches that may be considered to restore the normal bronchial microbiota are low-dose long-term antibiotic treatment and bacteriotherapy using the model promoted for gut microbiota.

Long-term macrolide antibiotics may be helpful due to their ability to regulate the altered microbiome, due to their antineutrophilic potential (decreased IL-8 production, neutrophil migration and/or function), or due to antiviral actions and the ability to restore corticosteroid sensitivity [59]. 
Predicting response to macrolides is still difficult. In one trial, responders were older and had a longer duration of asthma [60]. Other studies have suggested that PCR identification of Mycoplasma and Chlamydophila pneumoniae or neutrophilic inflammation best identifies the macrolide responsive phenotype $[61,62]$. In the AZISAST study, subjects with exacerbation-prone severe asthma received low-dose azithromycin or placebo as addon treatment to combination therapy of ICS and LABA for 6 months. While there was no difference between groups in the rate of severe exacerbations and lower respiratory tract infections requiring treatment with antibiotics, there was a significant difference in the predefined subgroup of subjects with non-eosinophilic severe asthma. There were no significant effects on lung function. Azithromycin was well-tolerated but was associated with increased oropharyngeal carriage of macrolide-resistant streptococci [63•].

\section{Obese asthma phenotype}

Analysis of the British Thoracic Society Difficult Asthma Registry Patient cohort according to BMI suggests that obesity-associated severe asthma represents a distinct clinical phenotype with greater requirements for maintenance corticosteroid, steroid burst therapy, and reliever use per day. These patients have shown reduced FVC and elevated carbon monoxide transfer coefficient. Serum IgE levels decreased with increasing BMI, and the obese group was more likely to report eczema but less likely to have a history of nasal polyps [64].

Excess BMI is an established risk factor for asthma, particularly in women. While it has been hypothesized that the metabolic syndrome mediates the BMI-asthma association, the CARDIA study found that BMI was a stronger predictor than metabolic syndrome for incident asthma in women. These results suggest that the BMI-asthma association is attributable to biomechanical, metabolic, or inflammatory abnormalities associated with obesity that are not part of the metabolic syndrome [65•]. In a cross-sectional comparison between early- and late-onset asthma phenotypes in the Severe Asthma Research Program, subjects with late-onset asthma had a higher median plasma asymmetric dimethyl arginine (ADMA) level and lower median plasma L-arginine. The log of plasma L-arginine/ADMA was inversely correlated with BMI and was associated with less IgE, increased respiratory symptoms, lower lung volumes, and worse QoL. The authors suggest abnormal metabolic pathways in obese asthma featuring an ADMA-driven impairment in protective NO synthesis. This obese asthma endotype is particularly relevant for patients in whom inflammation is not predominant and who are less responsive to anti-inflammatory strategies [66•]. In 131 subjects with severe asthma categorized into lean, overweight, and obese groups defined by their BMI, sputum IL-5 geometric mean was elevated in the obese compared with overweight and lean subjects and was correlated with BMI. In the bronchoscopy group, the submucosal eosinophil number was correlated with BMI, and the median number of submucosal eosinophils was higher in obese versus lean subjects. As this was a single-center study, further studies 
replicating these results are needed, but this may suggest a subset of obese asthmatics where specific antieosinophilic therapy is beneficial [67].

\title{
Compliance with Ethics Guidelines
}

\author{
Conflict of Interest \\ Ioana Octavia Agache declares no conflict of interest.
}

Human and Animal Rights and Informed Consent

This article does not contain any studies with human or animal subjects performed by any of the authors.

\section{References and Recommended Reading}

Papers of particular interest, published recently, have been highlighted as:

- Of importance

$\bullet \quad$ Of major importance

1. Bacci E, Cianchetti S, Bartoli M, Dente FL, Di Franco A, Vagaggini B, et al. Low sputum eosinophils predict the lack of response to beclomethasone in symptomatic asthmatic patients. Chest. 2006;129:565-72. 9.

2. Green RH, Brightling CE, McKenna S, Hargadon B, Parker D, Bradding P, et al. Asthma exacerbations and sputum eosinophil counts: a randomised controlled trial. Lancet. 2002;360:1715-21.

3. Lex C, Jenkins G, Wilson NM, Zacharasiewicz A, Erin E, Hansel TT, et al. Does sputum eosinophilia predict the response to systemic corticosteroids in children with difficult asthma? Pediatr Pulmonol. 2007;42:298-303.

4. Martin RJ, Szefler SJ, King TS, Kraft M, Boushey HA, Chinchilli VM, et al. The Predicting Response to Inhaled Corticosteroid Efficacy (PRICE) trial. J Allergy Clin Immunol. 2007;119:73-80.

5. Meijer RJ, Postma DS, Kauffman HF, Arends LR, Koëter GH, Kerstjens HA. Accuracy of eosinophils and eosinophil cationic protein to predict steroid improvement in asthma. Clin Exp Allergy. 2002;32(7):1096-103.

6. Wang F, He XY, Baines KJ, Gunawardhana LP, Simpson JL, Li F, et al. Different inflammatory phenotypes in adults and children with acute asthma. Eur Respir J. 2011;38:567-74.

7. Fleming L, Tsartsali L, Wilson N, Regamey N, Bush A. Sputum inflammatory phenotypes are not stable in children with asthma. Thorax. 2012;67(8):675-81.

8. Petsky HL, Cates CJ, Li A, Kynaston JA, Turner C, Chang AB. Tailored interventions based on exhaled nitric oxide versus clinical symptoms for asthma in children and adults. Cochrane Database Syst Rev. 2009;4, CD006340.

9. Pijnenburg MW, De Jongste JC. Exhaled nitric oxide in childhood asthma: a review. Clin Exp Allergy. 2008;38(2):246-59.

10. Lex C, Ferreira F, Zacharasiewicz A, Nicholson AG, Haslam PL, Wilson NM, et al. Airway eosinophilia in children with severe asthma: predictive values of noninvasive tests. Am J Respir Crit Care Med. 2006; 174:1286-91.

11. Hastie AT, Moore WC, Li H, Rector BM, Ortega VE, Pascual RM, et al. Biomarker surrogates do not accurately predict sputum eosinophil and neutrophil percentages in asthmatic subjects. J Allergy Clin Immunol. 2013;132:72-80.

12. Fleming L, Tsartsali L, Wilson N, Regamey N, Bush A. Longitudinal relationship between sputum eosinophils and exhaled nitric oxide in children with asthma. Am J Respir Crit Care Med. 2013;188(3):400-2.

13. Malinovschi A, Fonseca JA, Jacinto T, Alving K, Janson C. Exhaled nitric oxide levels and blood eosinophil counts independently associate with wheeze and asthma events in National Health and Nutrition Examination Survey subjects. J Allergy Clin Immunol. 2013;132:821-7.

The study shows that blood eosinophils and FeNO are independent predictors for asthma events and response to treatment supporting the 2 main subendotypes of Th- 2 inflammation. 
14.• Pavord ID, Korn S, Howarth P, Bleecker ER, Buhl R, Keene ON, et al. Mepolizumab for severe eosinophilic asthma (DREAM): a multicentre, doubleblind, placebo-controlled trial. Lancet. 2012;380(9842):651-9.

DREAM is the first large randomized study validating the endotype-driven approach in severe asthma as a method to increase response to targeted treatment (anti IL-5). Patients were selected based on a composite measure of eosinophilic inflammation.

15.• Corren J, Lemanske RF, Hanania NA, Korenblat PE, Parsey MV, Arron JR, et al. Lebrikizumab treatment in adults with asthma. N Engl J Med. 2011;365(12):1088-98.

MILLY is one of the first targeted treatments in asthma highlighting the value of a biomarker used as a pathwayspecific diagnostic test.

16. van der Schee MP, Palmay R, Cowan JO, Taylor DR. Predicting steroid responsiveness in patients with asthma using exhaled breath profiling. Clin Exp Allergy. 2013;43(11):1217-25.

17. Agache IO. From phenotypes to endotypes to asthma treatment. Curr Opin Allergy Clin Immunol. 2013;13(3):249-56.

The review critically revises new approaches to classify asthma and the emerging endotype-driven strategies.

18. Agache I, Akdis C, Jutel M, Virchow JC. Untangling asthma phenotypes and endotypes. Allergy. 2012;67(7):835-46.

Comprehensive description of asthma phenotypes and corresponding endotypes grading the level of evidence for corresponding biomarkers.

19.• Lötvall J, Akdis CA, Bacharier LB, Bjermer L, Casale TB, Custovic A, et al. Asthma endotypes: a new approach to classification of disease entities within the asthma syndrome. J Allergy Clin Immunol. 2011;127(2):355-60.

The PRACTAAL consensus proposed the criteria to validate an asthma endotype.

20. Woodruff PG, Modrek B, Choy DF, Jia G, Abbas AR, Ellwanger A, et al. T-helper type 2-driven inflammation defines major subphenotypes of asthma. Am J Respir Crit Care Med. 2009;180(5):388-95.

21. Bhakta NR, Solberg OD, Nguyen CP, Nguyen CN, Arron JR, Fahy JV, et al. A qPCR-based metric of Th2 airway inflammation in asthma. Clin Transl Allergy. 2013;3(1):24.

22. Peters MC, Mekonnen ZK, Yuan S, Bhakta NR, Woodruff PG, Fahy JV. Measures of gene expression in sputum cells can identify TH2-high and TH2-low subtypes of asthma. J Allergy Clin Immunol. 2013. doi:10.1016/j.jaci.2013.07.036.

23. Busse WW, Morgan WJ, Gergen PJ, Mitchell HE, Gern JE, Liu AH, et al. Randomized trial of omalizumab (anti-IgE) for asthma in inner-city children. N Engl J Med. 2011;364:1005-15.

24. Hanania NA, Wenzel S, Rosén K, Hsieh HJ, Mosesova $\mathrm{S}$, Choy DF, et al. Exploring the effects of omalizumab in allergic asthma: an analysis of biomarkers in the EXTRA study. Am J Respir Crit Care Med. 2013;187:804-11.

First study to validate a composite biomarker measure for effectiveness of omalizumab in severe allergic asthma.

25. Garcia G, Magnan A, Chiron R, Contin-Bordes C, Berger $\mathrm{P}$, Taillé $\mathrm{C}$, et al. A proof-of-concept, randomized, controlled trial of omalizumab in patients with severe, difficult-to-control, nonatopic asthma. Chest. 2013;144:411-9.

Proof-of-concept study highlighting the potential use of anti IgE targeted treatment in non-atopic asthma together with the putative mechanism of action.

26. Gill MA, Bajwa G, George TA, Dong CC, Dougherty II, Jiang $\mathrm{N}$, et al. Counterregulation between the FcepsilonRI pathway and antiviral responses in human plasmacytoid dendritic cells. J Immunol. 2010;184:5999-6006.

27. Mouthuy J, Detry B, Sohy C, Pirson F, Pilette C. Presence in sputum of functional dust mite-specific IgE antibodies in intrinsic asthma. Am J Respir Crit Care Med. 2011;184:206-14.

28. Gevaert P, Calus L, Van Zele T, Blomme K, De Ruyck $\mathrm{N}$, Bauters $\mathrm{W}$, et al. Omalizumab is effective in allergic and nonallergic patients with nasal polyps and asthma. J Allergy Clin Immunol. 2013;131:110-6.

29. McClintock D, Corren J, Hanania N. Lebrikizumab, an anti-IL-13 monoclonal antibody, reduces severe asthma exacerbations over 32 weeks in adults with inadequately controlled asthma. Am J Respir Crit Care Med. 2012;185:A3959.

30. Noonan M, Korenblat P, Mosesova S, Scheerens H, Arron JR, Zheng Y, et al. Dose-ranging study of lebrikizumab in asthmatic patients not receiving inhaled steroids. J Allergy Clin Immunol. 2013;132:567-74.

31. Scheerens H, Arron JR, Zheng Y, Putnam WS, Erickson RW, Choy DF, et al. The effects of lebrikizumab in patients with mild asthma following whole lung allergen challenge. Clin Exp Allergy. 2014;44(1):38-46.

32.• Jia G, Erickson RW, Choy DF, Mosesova S, Wu LC, Solberg OD, et al. Periostin is a systemic biomarker of eosinophilic airway inflammation in asthmatic patients. J Allergy Clin Immunol. 2012;130(3):64754.

First study to prove periostin as a robust biomarker of eosinophilic inflammation in asthma.

33. Piper E, Brightling C, Niven R, Oh C, Faggioni R, Poon $\mathrm{K}$, et al. A phase 2 placebo-controlled study of tralokinumab in moderate-to-severe asthma. A Phase IIb, 52-week randomized, double-blind study to evaluate the efficacy (asthma exacerbations as primary end point) of tralokinumab in adults with uncontrolled, severe asthma is underway (NCT01402986). Eur Respir J. 2013;41:330-8.

34. Slager RE, Otulana BA, Hawkins GA, Yen YP, Peters $\mathrm{SP}$, Wenzel SE, et al. IL-4 receptor polymorphisms 
predict reduction in asthma exacerbations during response to an anti-IL-4 receptor $\alpha$ antagonist. J Allergy Clin Immunol. 2012;130:516-22.

35. Wenzel S, Ford L, Pearlman D, Spector S, Sher L, Skobieranda F, et al. Dupilumab in persistent asthma with elevated eosinophil levels. N Engl J Med. 2013;368(26):2455-66.

First large randomized trial evaluating the safety and efficacy of anti-IL-4 targeted interventions in eosinophilic asthma.

36. Haldar P, Brightling CE, Hargadon B, Gupta S, Monteiro W, Sousa A, et al. Mepolizumab and exacerbations of refractory eosinophilic asthma. N Engl J Med. 2009;360:973-84.

37. Nair P, Pizzichini MM, Kjarsgaard M, Inman MD, Efthimiadis A, Pizzichini E, et al. Mepolizumab for prednisone-dependent asthma with sputum eosinophilia. N Engl J Med. 2009;360:985-93.

38. Castro M, Mathur S, Hargreave F, Boulet LP, Xie F, Young J, et al. Res-5-0010 Study Group. Reslizumab for poorly controlled, eosinophilic asthma: a randomized, placebo-controlled study. Am J Respir Crit Care Med. 2011;184:1125-32.

39. Nair P, Gaga M, Zervas E, Alagha K, Hargreave FE, O'Byrne PM, et al. Safety and efficacy of a CXCR2 antagonist in patients with severe asthma and sputum neutrophils: a randomized, placebo-controlled clinical trial. Clin Exp Allergy. 2012;42:1097-103.

40. Busse WW, Holgate S, Kerwin E, Chon Y, Feng J, Lin J, et al. Randomized, double-blind, placebo-controlled study of brodalumab, a human anti-IL-17 receptor monoclonal antibody, in moderate to severe asthma. Am J Respir Crit Care Med. 2013;188:1294-302.

First large clinical trial to evaluate the efficacy and safety of anti-IL-17 targeted intervention in asthma and delineating a possible responder subgroup.

41. Holgate ST, Davies DE, Powell RM, Howarth PH, Haitchi HM, Holloway JW. Local genetic and environmental factors in asthma disease pathogenesis: chronicity and persistence mechanisms. Eur Respir J. 2007;29:793-803.

42. Gerthoffer WT, Solway J, Camoretti-Mercado B. Emerging targets for novel therapy of asthma. Curr Opin Pharmacol. 2013;13:324-30.

43. Deshpande DA, Wang WC, McIlmoyle EL, Robinett KS, Schillinger RM, An SS, et al. Bitter taste receptors on airway smooth muscle bronchodilate by localized calcium signaling and reverse obstruction. Nat Med. 2010;16:1299-304.

44. James A, Daham K, Dahlen B, Hedlin G, Kere J, Konradsen J, et al. Expression of bitter taste receptors on peripheral blood leukocytes from asthmatic patients. Am J Respir Crit Care Med. 2012;185:A6752.

45. Orsmark-Pietras C, James A, Konradsen JR, Nordlund B, Soderhall C, Pulkkinen V, et al. Transcriptome analysis reveals up-regulation of bitter taste receptors in severe asthmatics. Eur Respir J. 2013;42:65-78.
46. An SS, Wang WC, Koziol-White CJ, Ahn K, Lee DY, Kurten RC, et al. TAS2R activation promotes airway smooth muscle relaxation despite beta (2)-adrenergic receptor tachyphylaxis. Am J Physiol Lung Cell Mol Physiol. 2012;303:L304-11.

47. Thomas B, Rutman A, Hirst RA, Haldar P, Wardlaw AJ, Bankart J, et al. Ciliary dysfunction and ultrastructural abnormalities are features of severe asthma. J Allergy Clin Immunol. 2010;126:722-9.

48. Hasani A, Toms N, O'Connor J, Dilworth JP, Agnew JE. Effect of salmeterol xinafoate on lung mucociliary clearance in patients with asthma. Respir Med. 2003;97:667-71.

49. Liu YJ. Thymic stromal lymphopoietin and OX40 ligand pathway in the initiation of dendritic cell-mediated allergic inflammation. J Allergy Clin Immunol. 2007;120:238-44.

50. Zhang F, Huang G, Hu B, Song Y, Shi Y. A soluble thymic stromal lymphopoietin (TSLP) antagonist, TSLPR-immunoglobulin, reduces the severity of allergic disease by regulating pulmonary dendritic cells. Clin Exp Immunol. 2011;164:256-64.

51. Bochkov YA, Busse WW, Brockman-Schneider RA, Evans MD, Jarjour NN, McCrae C, et al. Budesonide and formoterol effects on rhinovirus replication and epithelial cell cytokine responses. Respir Res. 2013;14:98.

52. Sykes A, Edwards MR, Macintyre J, del Rosario A, Bakhsoliani E, Trujillo-Torralbo $\mathrm{MB}$, et al. Rhinovirus 16 -induced IFN- $\alpha$ and IFN- $\beta$ are deficient in bronchoalveolar lavage cells in asthmatic patients. J Allergy Clin Immunol. 2012;129:1506-14.e6.

53. Wark PA, Johnston SL, Bucchieri F, Powell R, Puddicombe S, Laza-Stanca V, et al. Asthmatic bronchial epithelial cells have a deficient innate immune response to infection with rhinovirus. J Exp Med. 2005;201:937-47.

54. Djukanovic R, Harrison T, Monk P, Thomson N, Johnston S, Holgate S, et al. The effects of interferon beta on cold-induced asthma exacerbations. ERS 2012, abstract number 347.

55. Han MK, Huang YJ, Lipuma JJ, Boushey HA, Boucher $\mathrm{RC}$, Cookson WO, et al. Significance of the microbiome in obstructive lung disease. Thorax. 2012;67:456-63.

56. Hilty $M$, Burke C, Pedro H, Cardenas P, Bush A, Bossley C, et al. Disordered microbial communities in asthmatic airways. PLoS ONE. 2010;5:e8578.

57. Huang YJ, Nelson CE, Brodie EL, Desantis TZ, Baek MS, Liu J, et al. Airway microbiota and bronchial hyperresponsiveness in patients with suboptimally controlled asthma. J Allergy Clin Immunol. 2011;127:372-81.

58. Goleva E, Jackson LP, Harris JK, Robertson CE, Sutherland ER, Hall CF, et al. The effects of airway 
microbiome on corticosteroid responsiveness in asthma. Am J Respir Crit Care. 2013;188:1193-201. The study relates disturbed airway microbiome to corticosteroid-resistance and describes the putative mechanisms.

59. Cameron EJ, McSharry C, Chaudhuri R, Farrow S, Thomson NC. Long-term macrolide treatment of chronic inflammatory airway diseases: risks, benefits and future developments. Clin Exp Allergy.

2012;42:1302-12.

60. Coeman M, van Durme Y, Bauters F, Deschepper E, Demedts I, Smeets P, et al. Neomacrolides in the treatment of patients with severe asthma and/or bronchiectasis: a retrospective observational study. Ther Adv Respir Dis. 2011;5:377-86.

61. Sutherland ER, King TS, Icitovic N, Ameredes BT, Bleecker E, Boushey HA, et al. A trial of clarithromycin for the treatment of suboptimally controlled asthma. J Allergy Clin Immunol. 2010;126:747-53.

62. Simpson JL, Powell H, Boyle MJ, Scott RJ, Gibson PG. Clarithromycin targets neutrophilic airway inflammation in refractory asthma. Am J Respir Crit Care Med. 2008; 177:148-55.

63. Brusselle GG, Vanderstichele C, Jordens P, Deman $\mathrm{R}$, Slabbynck H, Ringoet $\mathrm{V}$, et al. Azithromycin for prevention of exacerbations in severe asthma (AZISAST): a multicentre randomised doubleblind placebo-controlled trial. Thorax. 2013;68(4):322-9.
First low-dose macrolide large trial in exacerbation-prone severe asthma highlighting the non-eosinophilic asthma responders group.

64. Gibeon D, Batuwita K, Osmond M, Heaney LG, Brightling CE, Niven R, et al. Obesity-associated severe asthma represents a distinct clinical phenotype: analysis of the British Thoracic Society Difficult Asthma Registry Patient cohort according to BMI. Chest. 2013;143:406-14.

65. Assad N, Qualls C, Smith LJ, Arynchyn A, Thyagarajan $\mathrm{B}$, Schuyler $\mathrm{M}$, et al. Body mass index is a stronger predictor than the metabolic syndrome for future asthma in women. The longitudinal CARDIA study. Am J Respir Crit Care Med. 2013;188(3):319-26.

Results from CARDIA asthma substudy highlight the role of inflammatory and metabolic pathways independent of the metabolic syndrome in obese asthma.

66. Holguin F, Comhair SA, Hazen SL, Powers RW, Khatri SS, Bleecker ER, et al. An association between L-arginine/asymmetric dimethyl arginine balance, obesity, and the age of asthma onset phenotype. Am J Respir Crit Care Med. 2013;187(2):153-9.

The study advances a new hypothesis of metabolic disturbed pathway in obese asthma.

67. Desai D, Newby C, Symon FA, Haldar P, Shah S, Gupta S, et al. Elevated sputum interleukin-5 and submucosal eosinophilia in obese individuals with severe asthma. Am J Respir Crit Care Med. 2013;188(6):657-63. 